Review Article

\title{
Mechanisms of Oxidative Stress and Therapeutic Targets following Intracerebral Hemorrhage
}

\author{
Zhenjia Yao, ${ }^{1}$ Qinqin Bai, ${ }^{1}$ and Gaiqing Wang $\mathbb{D D}^{1,2}$ \\ ${ }^{1}$ Neurology, Shanxi Medical University, 030001 Taiyuan, Shanxi, China \\ ${ }^{2}$ Neurology, Sanya Central Hospital (Hainan Third People’s Hospital), 572000 Sanya, Hainan, China \\ Correspondence should be addressed to Gaiqing Wang; wanggq08@163.com
}

Received 29 September 2020; Revised 17 January 2021; Accepted 10 February 2021; Published 22 February 2021

Academic Editor: John H. Zhang

Copyright (C) 2021 Zhenjia Yao et al. This is an open access article distributed under the Creative Commons Attribution License, which permits unrestricted use, distribution, and reproduction in any medium, provided the original work is properly cited.

Oxidative stress (OS) is induced by the accumulation of reactive oxygen species (ROS) following intracerebral hemorrhage (ICH) and plays an important role in secondary brain injury caused by the inflammatory response, apoptosis, autophagy, and blood-brain barrier (BBB) disruption. This review summarizes the current state of knowledge regarding the pathogenic mechanisms of brain injury after $\mathrm{ICH}$, markers for detecting OS, and therapeutic strategies that target OS to mitigate brain injury.

\section{Introduction}

ICH is a type of stroke characterized by spontaneous and nontraumatic bleeding in the brain that is associated with high morbidity and mortality rates [1]. ICH can be classified as primary and secondary. While treatment options for the former are limited, various strategies have been proposed for managing the latter [1].

Hematomal and perihematomal regions are biochemically active environments that sustain oxidative damage following ICH [2]. OS is defined as an imbalance between the formation of strong oxidants and physiologic antioxidant capacity [3]. ROS such as oxygen free radicals (e.g., superoxide $\left(\mathrm{O}_{2}^{-}\right)$and hydroxyl radicals $\left.\left(\mathrm{OH}^{-}\right)\right)$and nonradical compounds (e.g., hydrogen peroxide $\left(\mathrm{H}_{2} \mathrm{O}_{2}\right)$ and hypochlorous acid), as well as reactive nitrogen species (RNS; e.g., nitric oxide (NO)) and a variety of nitrogenous compounds produced as metabolic byproducts, are the major drivers of oxidative damage [4] to proteins, lipids, and nucleic acids, which can induce inflammation, autophagy, apoptosis, and destruction of the BBB. OS is associated with dysregulation of cellular oxidation and reduction (redox) mechanisms; redoxsensitive thiols that are easily oxidized by nonradical oxidants such as $\mathrm{H}_{2} \mathrm{O}_{2}$ after ICH are essential for transcription factor regulation (e.g., nuclear factor erythroid 2-related factor (Nrf) 2 and nuclear factor- (NF-) $\kappa \mathrm{B}$ ) [5].

The Kelch-like ECH-associated protein (Keap) 1/Nrf2/antioxidant response element (ARE) signaling pathway is the main regulatory system protecting cells against oxidative damage. Nrf2 is a master regulator of the cellular response to oxidative stress, which is associated with the expression of antioxidant and detoxification enzymes and factors such as $\mathrm{NAD}(\mathrm{P}) \mathrm{H}$ : quinone oxidoreductase (NQO) 1, catalase (CAT), superoxide dismutase (SOD), heme oxygenase- (HO-) 1, glutathione peroxidase (GPX), and glutathione-S-transferase (GST) [6]. Nrf2 was shown to mitigate early brain injury after ICH by translocating to the nucleus following activation and binding to AREs to activate the transcription of genes encoding antioxidant enzymes [7].

\section{ROS Production after ICH}

2.1. Production of ROS by Activated Phagocytes and Nonphagocytic Cells following ICH. Activated neutrophils, microglia, and macrophages are the main sources of ROS following ICH. Nicotinamide adenine dinucleotide phosphate (NADPH) oxidase (NOX) is expressed on the surface of neutrophils and macrophages and stimulates the production of 
ROS in response to extracellular signals such as hormones and cytokines [8]. Nonphagocytic cells such as neurons, microglia, astrocytes, and cerebrovascular endothelial cells also express NOX [8-10]. To date, 7 NOX isozymes have been identified in nonphagocytic cells that use NADH or NADPH as an electron donor for ROS production [11]. While NOX activity is generally low in these cells, they continuously produce $\mathrm{O}_{2}{ }^{-}$even in the absence of external stimulation [11]. Hypoxia after $\mathrm{ICH}$ induces conformational changes in gp91phox, the heme-binding subunit of NOX, which activates the protein and leads to the formation of NOX complexes and increased ROS production [12] The activation of NOX was also reported to be the main mechanism underlying ROS generation in a rabbit model of intraventricular hemorrhage (IVH) [13], and OS resulting from NOX activation was shown to contribute to collagenaseinduced ICH and brain injury [14]. NOX2 protein level was upregulated in the striatum of mice $12 \mathrm{~h}$ after $\mathrm{ICH}$, which peaked at $24 \mathrm{~h} \mathrm{[15],} \mathrm{and} \mathrm{another} \mathrm{study} \mathrm{found} \mathrm{that} \mathrm{gp91phox}$ was primarily expressed in activated microglia and colocalized with peroxynitrite (ONOO-) $24 \mathrm{~h}$ after $\mathrm{ICH}$ in the injured hemisphere [16]. However, following $\mathrm{ICH}$, activated leukocytes release myeloperoxidase (MPO), which catalyzes lipid peroxidation and causes OS at the site of injury [17]. Additionally, increased expression of inducible NO synthase (iNOS) in M1 microglia in conjunction with the release of proinflammatory mediators and cytotoxic substances caused significant tissue damage after ICH [18].

\subsection{Increased ROS Production in Mitochondria following} $\mathrm{ICH}$. Another important ROS is $\mathrm{O}_{2}{ }^{-}$produced by mitochondria, which is generated as a byproduct of biological oxidation during mitochondrial respiration under physiologic conditions [19]. In most cells, the electron transport chain consumes $90 \%$ of cellular oxygen; $2 \%$ of this is transformed into oxygen free radicals in the mitochondrial inner membrane and matrix $[20,21]$. Electrons that leak from the respiratory chain react with oxygen to form $\mathrm{O}_{2}{ }^{-}$. Mitochondrial $\mathrm{O}_{2}{ }^{-}$is detoxified to $\mathrm{H}_{2} \mathrm{O}_{2}$ by SOD, then to $\mathrm{O}_{2}$ and $\mathrm{H}_{2} \mathrm{O}$ by antioxidant enzymes such as CAT and GPX. However, $\mathrm{O}_{2}{ }^{-}$ that elude antioxidant mechanisms can damage proteins, lipids, and DNA [21]. There are 7 known sources of $\mathrm{O}_{2}{ }^{-}$in mammalian mitochondria: the ubiquinone-binding sites in complex I (site IQ) and complex III (site IIIQo), glycerol 3phosphate dehydrogenase, complex I flavin (site IF), electron-transferring flavoprotein: $Q$ oxidoreductase in fatty acid beta oxidation, pyruvate, and 2-oxoglutarate dehydrogenase, with site IQ and site IIIQo having the highest production capacities [21].

Mitochondria are storage sites for calcium ions $\left(\mathrm{Ca}^{2+}\right)$. Under ischemia/reperfusion (I/R), excessive glutamate levels can cause an influx of $\mathrm{Ca}^{2+}$ into neurons via $\mathrm{N}$-methyl-daspartic acid receptor (NMDAR), a ligand-gated ion channel [22]. Activation of the NMDAR leads to further $\mathrm{Ca}^{2+}$ influx, with increased levels in the cytosol and mitochondrial $\mathrm{Ca}^{2+}$ loading. Thrombin produced after ICH leads to Src kinase activation by activating protease-activated receptor 1 (PAR1), which phosphorylates and activates NMDAR. PARs are a subfamily of $G$ protein-coupled receptors (GPCRs) with four members, namely, PAR1, PAR2, PAR3, and PAR4. PAR1 is highly expressed in many different cell types. PAR1 plays an important role in astrocyte proliferation, stimulus-induced long-term potentiation (LTP), and nerve growth factor (NGF) secretion. PAR1 enhances Srcmediated tyrosine phosphorylation of NMDA receptor in ICH [23]. Activation of $\alpha$-amino-3-hydroxy-5-methyl-4-isoxazolepropionic acid (AMPA) receptor by glutamate after $\mathrm{ICH}$ in motor neurons also increased $\mathrm{Ca}^{2+}$ and $\mathrm{Na}+$ influx and mitochondrial $\mathrm{Ca}^{2+}$ loading [22]. Following $\mathrm{ICH}, \mathrm{Ca}^{2+}$ stored in the endoplasmic reticulum (ER) is thought to be sequestered by mitochondria. Mitochondrial $\mathrm{Ca}^{2+}$ loading reduces mitochondrial membrane potential (MMP) and opens the mitochondrial permeability transition pore (MPTP), resulting in mitochondrial damage and disruption of the mitochondrial respiratory chain; together, these processes result in the release of excess ROS [22].

2.3. Increased ROS Production by the ER following ICH. The ER is the site of protein synthesis, posttranslational modification, folding, and trafficking. ICH can cause ER stress (ERS), which is characterized by protein misfolding, accumulation of abnormal proteins, and $\mathrm{Ca}^{2+}$ imbalance, all of which trigger the unfolded protein response (UPR) [24]. Glutamate excitotoxicity and the inflammatory response can result in ERS. When ICH causes ERS, ROS are generated by NOX4 in the internal membrane. ROS then acts as a signaling intermediate that subsequently mitigates ERS via the UPR. If ERS is not alleviated, the delayed expression of proteins such as $\mathrm{C} / \mathrm{EBP}$ homologous protein (CHOP) causes a secondary increase in ROS levels [25]. Additionally, disulfide bonds in proteins translated in the ER are highly sensitive to changes in redox balance; thus, both reducing and oxidizing conditions can disrupt protein folding and cause ERS. On the other hand, oxidative protein folding is a major source of intracellular ROS production [26]; during this process, thiol groups on the cysteines of peptides are oxidized and form disulfide bonds [26]. After accepting electrons from protein disulfide isomerase (PDI), ER oxidoreductin (ERO) 1 transfers electrons to molecular oxygen to generate $\mathrm{H}_{2} \mathrm{O}_{2}$, the major type of ROS formed in the ER lumen [26]. In the ERS following $\mathrm{ICH}$, disruption of disulfide bond formation leads to ROS accumulation and OS [26]. Inositol 1,4,5-trisphosphate receptor and voltage-dependent anion channel-which are located in the ER and mitochondria, respectively-form a complex with the chaperone protein glucose-regulated protein (GRP) 75, thus physically connecting the 2 organelles [22]. Upon ERS, $\mathrm{Ca}^{2+}$ transfer at the contact points between the ER and mitochondria leads to mitochondrial dysfunction, thereby increasing mitochondrial ROS production, resulting in cellular stress or neuronal death. Although there have been few studies investigating the relationship between ERS and ICH, given the interaction between ERS and microglial activation [27], neuroinflammation, and autophagy after $\mathrm{ICH}$, clarification of this point can inspire new avenues for ICH treatment.

2.4. Hemoglobin $(\mathrm{Hb})$ Toxicity after $\mathrm{ICH}$. $\mathrm{Hb}$ toxicity is induced by free radicals generated via Fenton-type reactions 
and by oxidative damage to proteins, nucleic acids, and lipids [28]. During its conversion to methemoglobin, oxyhemoglobin releases $\mathrm{O}_{2}{ }^{-}$, which in turn forms $\mathrm{OH}-$ and contributes to ROS production [29]. Hb, a major component of erythrocytes, is a heterotetramer composed of $\alpha$ and $\beta$ globin subunits that each bind a heme molecule. $\mathrm{Hb}$ induces the expression of iNOS by M1 microglia and neutrophils after ICH. NOS is expressed by endothelial cells, macrophages, neurophagocytes, and nerve cells; there are 2 isoenzymes besides iNOS-namely, neural and endothelial NOS [30]. Overexpression of iNOS or endothelial NOS and the consequent overproduction of $\mathrm{NO}$ lead to changes in tight junction proteins and can potentially disrupt the BBB [31]. Following $\mathrm{ICH}$, heme is released by $\mathrm{Hb}$ and decomposed into bilirubin, free iron, and carbon monoxide.

2.5. Increased ROS Production by Heme following ICH. Heme (ferrous protoporphyrin IX) is a reactive, low molecular weight form of iron that participates in Fenton-type oxygen radical reactions in neurons, microglia, and neutrophils [32]. Hemin, the oxidized form of heme, accumulates in intracranial hematomas and is a potent oxidant [33]. Hemin is bound by hemopexin in serum, and the complex is translocated into the cell via lipoprotein receptor-related protein (LRP) 1. Intracellular hemin is degraded into bilirubin, $\mathrm{Fe}^{2+}$, and carbon monoxide. $\mathrm{Fe}^{2+}$ derived from hemin can generate $\mathrm{OH}-$ - the most reactive oxygen radical-via the Fenton reaction, leading to an increase in ROS levels [22].

2.6. Increased ROS Production from Ferrous Iron and Ferritin following ICH. Ferrous iron is one of the main contributors to OS following $\mathrm{ICH}$. Free iron catalyzes the conversion of $\mathrm{O}_{2}{ }^{-}$ and $\mathrm{H}_{2} \mathrm{O}_{2}$ into $\mathrm{OH}-$ via the Fenton reaction while oxidizing iron from a divalent to a trivalent form [34]. Ferrous iron is transported into the cell through divalent metal transporter (DMT) 1 and into mitochondria by ATP-binding cassette(ABC-) 7 [22], resulting in OS. Ferritin functions as a source of iron in lipid peroxidation; the release of iron from ferritin is mediated by $\mathrm{O}_{2}{ }^{-}$[34] (Figure 1). Knowledge of the mechanisms and dynamics of ROS generation following ICH can guide the development of drugs for the treatment of ICH that act by mitigating OS.

\section{OS-Induced Brain Damage following ICH}

3.1. Organelle Damage by OS. Oxidative damage in DNA includes base modifications and hydrogen bond breakage [35]; in proteins, it can include amino acid modifications, peptide chain fractures, and protein cross-linking [36]. Free radicals cause cellular membrane damage by promoting lipid peroxidation (especially of polyunsaturated fatty acids); the binding of free radicals to membrane receptors also leads to the destruction of membrane integrity [37].

3.2. OS-Induced Autophagy in ICH. Autophagy is a cellular process for the clearance of damaged organelles and proteins that are misfolded or no longer required. Autophagy is triggered by ICH-induced OS [38]. Studies have indicated that superoxide is the major form of ROS regulating autophagy [39]. A hallmark of the process is the reversible conjugation of autophagy-related protein (Atg) 8 to the autophagosome membrane. Under conditions of serum starvation, ROS (especially $\mathrm{H}_{2} \mathrm{O}_{2}$ ) induces the inactivation of Atg4 during autophagosome formation, which promotes the lipidation of Atg8. As the autophagosome matures and fuses to a lysosome, the reduction in $\mathrm{H}_{2} \mathrm{O}_{2}$ levels promotes the activation of Atg 4 along with the delipidation and recycling of Atg8 [39]. The regulation of autophagy is closely related to p62/Keap1/Nrf2 redox signaling. The autophagy-related factor p62 binds to ubiquitinated protein aggregates, and its affinity for Keap1 is increased upon phosphorylation at Ser351 [40]. This induces the degradation of Keap1 via autophagy and releases Nrf2, which accumulates and translocates to the nucleus where it binds to AREs to activate the transcription of genes encoding antioxidant enzymes and p62 as well as other autophagy-related factors [40]. ROS produced as a result of $\mathrm{ICH}$ act on the mammalian target of rapamycin complex (mTORC) 1/UNC51-like kinase (ULK) 1 and AMP-activated protein kinase (AMPK)/ULK1 signaling pathways that regulate autophagy. AMPK activation and mTORC1 inhibition in response to ROS lead to ULK1 activation and induction of autophagy [40]. Activated ULK1 phosphorylates its interaction partners Atg13 and FAK family-interacting protein of $200 \mathrm{kDa}$ (FIP200), resulting in the activation of the class III phosphoinositide 3kinase (PI3K) complex via activating molecule in BECN1regulated autophagy (AMBRA) 1, thus initiating the nucleation of autophagosomes from the ER or mitochondria [40]. Some research data suggest that oxidative stress induces autophagy activation, which may make $\mathrm{ICH}$-induced brain injury disappear [41]. This may also reduce early brain damage in SAH [42]. However, others believe that under- or overactivation of autophagy may lead to cell damage and death. The potential role of selective autophagy in the clinical treatment of hemorrhagic stroke has been recognized. The mechanism of autophagy activation mediated by mitochondrial and ERS induced by OS after ICH remains to be studied.

3.3. Apoptosis Induced by OS following ICH. Severe OS and a high intracellular concentration of $\mathrm{Ca}^{2+}$ following $\mathrm{ICH}$ induces MPTP opening and a reduction in MMP, with subsequent release of cytochrome $\mathrm{C}$ and other proapoptotic proteins from the mitochondrial membrane into the cytosol, which activates the intrinsic neuronal apoptosis pathway in mitochondria [43]. After cerebral hemorrhage (ICH), oxidative stress leads to DNA and protein damage, which leads to neuronal apoptosis.

3.4. Matrix Metalloproteinase (MMP) Activation by OS after $\mathrm{ICH}$. Following $\mathrm{ICH}, \mathrm{O}_{2}{ }^{-}$and $\mathrm{NO}$ levels are increased as a result of endothelial NOS and iNOS activities, respectively. In the context of cerebral $\mathrm{I} / \mathrm{R}, \mathrm{NO}$ reacts with $\mathrm{O}_{2}{ }^{-}$produced by gp91phox to form ONOO-. The activation of MMP-9 and MMP-2 by ONOO- results in the degradation of the tight junction proteins claudin-5 and occludin as well as the extracellular matrix, leading to disruption of the BBB [44]. ONOO- induces other forms of cellular damage including protein oxidation, DNA damage, lipid peroxidation, tyrosine nitration, and mitochondrial dysfunction. Notably, tyrosine 


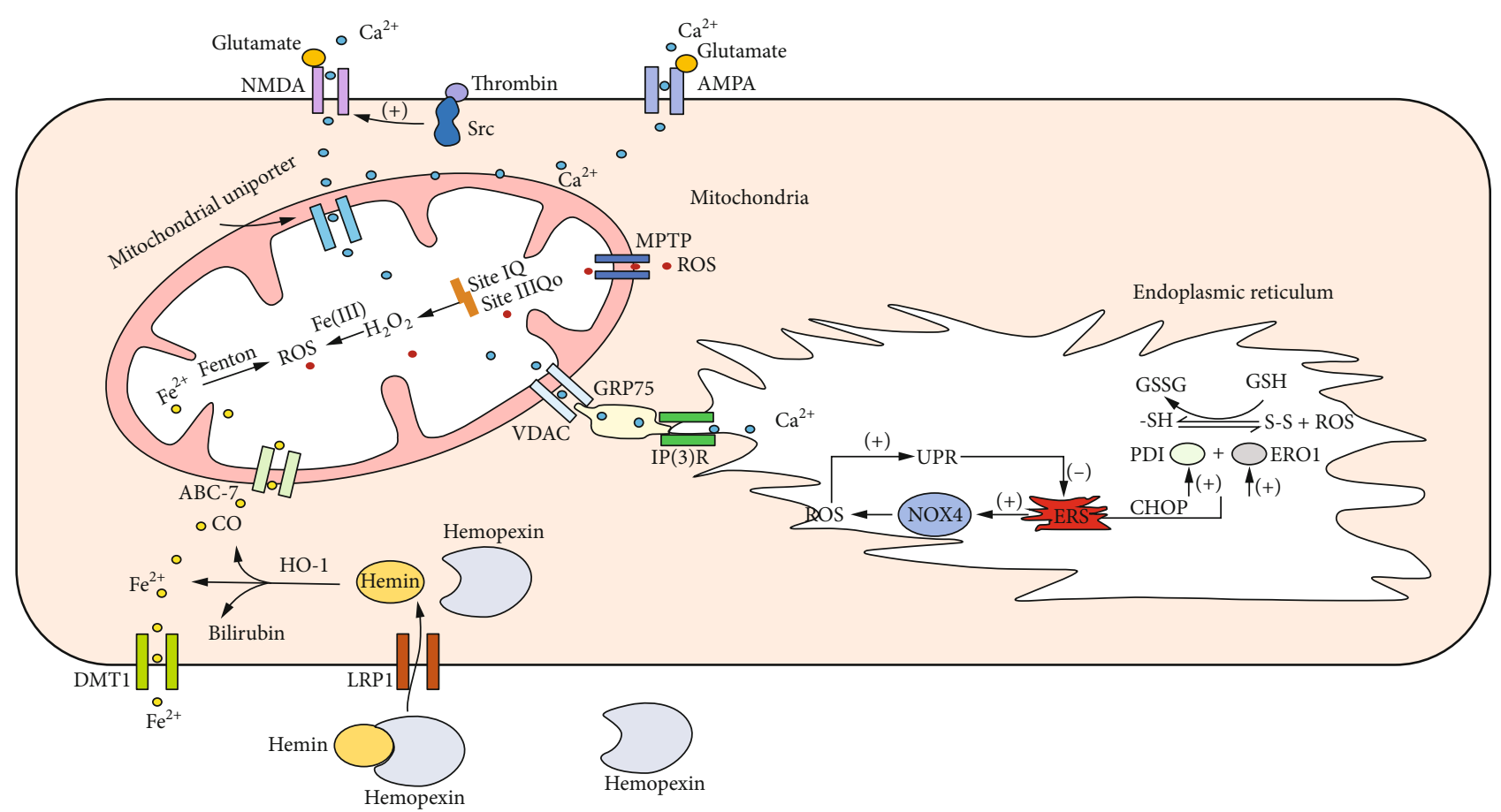

FIGURE 1: The production of reactive oxygen species after cerebral hemorrhage: hemin and divalent iron ions enter the cell through the corresponding receptors on the cell membrane, and further, Fenton reaction occurs in the mitochondria, thereby generating excess ROS; glutamate and thrombin activate ion channel receptors on the cell membrane to promote $\mathrm{Ca}^{2+}$ influx; in addition, the VDAC and IP(3) $\mathrm{R}$ located in the mitochondria and the endoplasmic reticulum are connected through GRP75, so that the $\mathrm{Ca}^{2+}$ in the endoplasmic reticulum flows into the mitochondria, and the $\mathrm{Ca}^{2+}$ in the mitochondria is further overloaded, and the MPTPA channel on the mitochondria is opened and released. The $\mathrm{H}_{2} \mathrm{O}_{2}$ produced at the two sites of site IQ and site IIIQo in the mitochondria generates ROS when it encounters $\mathrm{Fe}(\mathrm{III})$. Endoplasmic reticulum UPR can reduce ERS. When ERS cannot be relieved, certain UPR components (such as C/EBP homologous protein CHOP) may cause oxidative stress; in addition, in the stressed ER, the imbalance of disulfide bond formation and breaking may lead to the accumulation of reactive oxygen species (ROS) and cause oxidative stress. ROS: reactive oxygen species; Fe ${ }^{2+}$ : ferrous iron; AMPA: $\alpha$-amino-3-hydroxy-5-methyl-4-isoxazole-propionic acid receptor; NMDA: N-methyl-D-aspartic acid receptor; HO1: heme oxygenase-1; DMT: divalent metal transporter 1; MPTP: mitochondrial permeability transition pore; VDAC: voltage-dependent anion channel; ER: endoplasmic reticulum; IP3R: inositol 1,4,5-trisphosphate receptor; ERO1: ER oxidase 1; CHOP: C/EBP homologous protein; UPR: unfolded protein response; PDI: protein disulfide isomerases; LRP1: lipoprotein receptor-related protein; NOX4: adenine dinucleotide phosphate oxidase 4.

nitration leads to the modification of functional proteins and vascular endothelial cell injury [45]. Following ICH, Hbinduced OS resulting from MMP activation disrupted the $\mathrm{BBB}$ and induced cell apoptosis, which was reversed by overexpression of SOD1 [29].

3.5. OS Mediates the Inflammatory Response following ICH. ROS-induced activation of the NACHT, LRR, and PYD domain-containing protein (NLRP) 3 inflammasome following ICH results in the release of interleukin- (IL-) $1 \beta$, which promotes neutrophil infiltration, inflammation, and brain edema [46]. ROS and RNS produced by neutrophils regulate the inflammatory response after ICH by modulating phagocytosis, cellular function, gene expression, and apoptosis [47]. In the context of ICH, MPO from activated white blood cells catalyzes the oxidation of chloride ions by $\mathrm{H}_{2} \mathrm{O}_{2}$, producing the strong oxidant hypochlorous acid $(\mathrm{HOCl})$, which can cause further tissue damage and promote inflammation [48]. Thiol redox circuits are a normal part of cell signaling and physiologic regulation; their destruction in vascular disease causes OS, leading to the activation of a proinflamma- tory signaling cascade [5]. The net effect of NOX activation may be proinflammatory, as evidenced by its activation in phagocytes after ICH and the resultant generation of reactive oxygen intermediates that enhance inflammation and tissue damage [8] and further increase OS. The infiltration of inflammatory cells induces an influx of $\mathrm{Ca}^{2+}$, increasing free radical production, and lipid peroxidation. After ICH, M1 phenotypic microglia can be activated by thrombin to release proinflammatory cytokines and chemokines such as interleukin- $1 \beta$ (IL-1 $\beta$ ), tumor necrosis factor- $\alpha$ (TNF- $\alpha$ ), and ROS, thereby attracting surrounding inflammatory mediators. In addition, microglia strongly express $\mathrm{HO}-1$, which converts heme into iron, carbon monoxide (CO), and biliverdin [45] (Figure 2).

\section{Markers for Detecting OS after ICH}

\subsection{Oxidative DNA Damage}

4.1.1. 8-Oxo-7,8-Dihydro-2' Deoxyguanosine (8-OHdG) and 8-Oxo-7,8-Dihydro-Guanine (8-oxoGua). 8-OHdG and 8- 


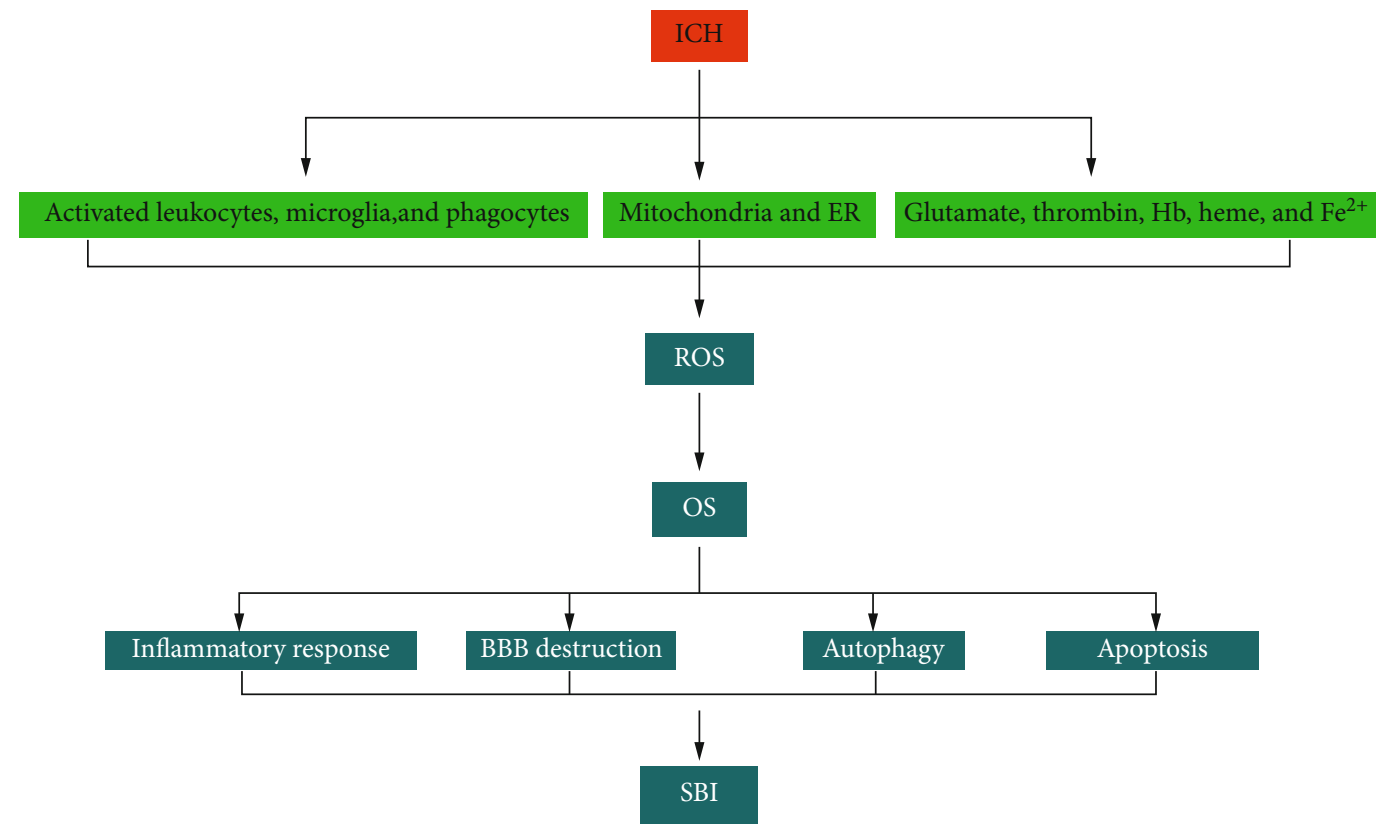

FIgURE 2: Summary of mechanisms by which OS aggravates SBI after ICH: after ICH, activated phagocytes, mitochondria, ER, and RBC lysates all cause excess release of ROS. This increase in OS exacerbates the inflammatory response, apoptosis, autophagy, and BBB disruption, with further SBI aggravation. BBB: blood-brain barrier; ICH: intracerebral hemorrhage; OS: oxidative stress; ROS: reactive oxygen species; SBI: secondary brain injury.

oxoGua are sensitive markers for DNA oxidative damage that are produced through hydroxylation at the C-8 position of guanine. Various methods are used to measure 8-oxoGua and 8-oxodG concentrations in blood and urine samples, including gas chromatography-mass spectrometry (GCMS), high-performance liquid chromatographyelectrochemical detection (HPLC-ECD), immunohistochemistry, and enzyme-linked immunosorbent assay (ELISA). GC-MS and HPLC-ECD have higher specificity and are more accurate than ELISA [49]. At $24 \mathrm{~h}$ after ICH, 8-OHdG was detected at high levels at the borders of damaged and normal tissues, coinciding with an increase in the number of apurinic/apyrimidinic sites; expression peaked in the first 3 days post-ICH and returned to baseline starting from day 7 [50]. The OS marker leukocyte 8-hydroxy-2 ${ }^{\prime}$ -deoxyguanosine was shown to be an independent predictor of 30-day outcome following ICH [51], while serum 8-OHdG and 8-oxoGua levels were related to 30-day mortality in ICH patients [52]. According to reports, the optimal cutoff of serum OGS levels in ICH patients is $4.94 \mathrm{ng} / \mathrm{mL}$ (according to Youden's $J$ index). When the OGS level of patients is higher than the cutoff value, the mortality rate is higher [52].

\subsection{Lipid Peroxidation}

4.2.1. Malondialdehyde (MDA). ICH leads to progressively higher levels of lipid peroxidation, as evidenced by increased diene conjugation and MDA levels [52]. MDA is the most commonly used biomarker of lipid peroxidation in clinical studies. Serum MDA levels were shown to increase rapidly at the early stage of ICH and were closely related to the severity of clinical symptoms [53]. MDA can be accurately detected by HPLC [53], whereas the detection of thiobarbituric acid-reactive substances by ultraviolet light (UV) spectrophotometry overestimated MDA levels in human plasma [43]. Serum MDA level at diagnosis of severe spontaneous ICH was shown to be associated with early mortality [54]. The study found that patients with spontaneous cerebral hemorrhage have a higher risk of death when the serum MDA is higher than $2.48 \mathrm{nmol} / \mathrm{mL}$ [54]. It is common to use the thiobarbituric acid reactive substance (TBARS) method to determine the serum MDA of cerebral hemorrhage mouse models and patients with ICH $[2,52,54-58]$.

4.2.2. 4-Hydroxynonenal (4-HNE). 4-HNE is a product of lipid peroxidation in the cell membrane that can be quantified by HPLC, GC-MS, LC-MS, and aldehyde-reactive probes [59]. 4-HNE levels in cerebrospinal fluid (CSF) and plasma samples of patients with Parkinson's disease have also been determined by GC-negative-ion chemical ionization mass spectrometry-based detection of O-pentafluorobenzyl oxime [60]. Vasospasm in patients with SAH has been evaluated based on measurement of lipid peroxidation; the levels of polyunsaturated fatty acid cyclization products, F2isoprostanes (F2-IsoPs), and neuroprostanes were highest on day 1 post-SAH and decreased over time, with similar trends observed in the levels of 4-HNE, 4-oxononenal, and MDA [61].

4.2.3. F2-IsoPs. Another hallmark of lipid peroxidation is the production of F2-IsoPs, which can be detected by LC-MS and the gold standard method GC-MS, while ELISA is less effective [62]. 8-Iso-prostaglandin (8-iso-PG) F2 $\alpha$ is an isomer derivative of F2-IsoP that serves as a biomarker for 
evaluating OS and lipid peroxidation. 8-Iso-PGF2 $\alpha$ is present in a free form in tissue or in esterified form in lipids; levels in the blood were shown to be increased after ICH, which was positively correlated with the National Institute of Health Stroke Scale score and hematoma volume [60], and elevated plasma concentrations of 8 -Iso-PGF $2 \alpha$ were reported to be associated with the clinical severity and outcome of $\mathrm{ICH}$. The plasma level of 8-iso-PGF $2 \alpha$ is an independent prognostic factor in $\mathrm{ICH}$ [63] and indicator of oxidation in analyses of F2-IsoP, isofuran, and F(4)-neuroprostane concentrations following aneurysmal SAH and traumatic brain injury. A higher level of F(4)-neuroprostanes in CSF more accurately reflects neural dysfunction than the elevated F2-IsoP level [64].

\subsection{Enzyme Activity}

4.3.1. SOD. Studies have determined that the level of SOD in patients with cerebral hemorrhage $(113.62 \pm 9.14 \mathrm{U} / \mathrm{mL})$ is significantly lower than healthy $(161.20 \pm 21.12 \mathrm{U} / \mathrm{mL})$ [65]. It is a common indicator for evaluating oxidative stress in mouse models of ICH $[2,6,56,58,66,67]$.

4.3.2. MPO. The activity of MPO, a potent oxidizing enzyme, can be determined by quantifying the level of 3-Cl-Tyr and the conversion of hydroethidine to 2-chloroethidium. The $\mathrm{MPO} / \mathrm{H}_{2} \mathrm{O}_{2}$ /chloride system of leukocyte activation is responsible for the generation of 3-Cl-Tyr, which is a biomarker of neuroinflammation [68]. Moreover, serum MPO concentration was found to be increased in ICH patients, which was correlated with ICH severity and prognosis [69]. In ICH animal experiments, MPO can be measured with immunofluorescence assay [55].

\subsection{Evaluation of Antioxidant Levels}

4.4.1. Glutathione (GSH) and Oxidized Glutathione (GSSG). The molar ratio of GSH to GSSG is a useful index of OS in ICH. Total GSH in cells is quantified by measuring the concentration of the glutathione-N-ethylmaleimide conjugate by UV/visible HPLC. However, under pathologic conditions, total GSH content is lower than that of GSSG. Spectrophotometry can be used to determine GSSG concentration by either the GSH recycling method or HPLC [70], while commercial GSH/GSSG detection kits are commonly used in studies involving ICH models $[6,57,66]$.

4.4.2. Allantoin. Allantoin is a physiologic antioxidant that can be detected in human plasma and serum samples by GC-MS [71]. It was also demonstrated that determination of urinary allantoin concentrations by GC-MS was useful for evaluating the efficacy of clinical interventions in preterm neonates diagnosed with germinal matrix IVH [64].

4.4.3. Thioredoxin (TRX). TRX is an antioxidant that eliminates oxygen as well as $\mathrm{OH}$ - radicals. Serum concentrations of TRX can be determined by ELISA, with commercial kits widely available. Increased serum concentrations of TRX were shown to be related to hemorrhage severity and longterm mortality in patients with ICH [72].
Besides, the mouse ICH model can also use the ROS analysis kit to detect the level of oxidative stress. This kit uses the principle of the fluorescent probe $2^{\prime}, 7^{\prime}$-dichlorofluorescein diacetate to determine $[6,57,67,73]$.

\section{Therapeutic Strategies Targeting OS following ICH (Supplementary Table 1-2)}

\subsection{Regulation of Oxidant Signaling Pathways}

5.1.1. Keap1/Nrf2/ARE Signaling (Supplementary Table 1). The Keap1/Nrf2/ARE signaling pathway is one of the most important defense mechanisms against OS in ICH [74] as it promotes the expression of endogenous antioxidant enzymes including NQO1, CAT, SOD, HO-1, and GPX [75]. Nrf2 was upregulated following $\mathrm{ICH}$, with peak level occurring at $24 \mathrm{~h}$; the time course of expression was shown to be correlated with the severity of brain edema and neurologic deficits. Heme oxygenase- 1 is resistant to OS in the early stages of $\mathrm{ICH}$ but is thought to promote oxidation in subsequent stages of the disease process. Drugs targeting the Nrf2/ARE signaling pathway have therapeutic potential for reducing brain damage caused by OS and inflammation following ICH (Figure 3). The drugs used to treat ICH animal models by regulating the Nrf2-ARE signaling pathway include glycyrrhizin [6], simvastatin [76], methyl hydrogen fumarate [77], nicotinamide mononucleotide [56], astaxanthin [55], mangiferin [71], RS9 [78], silymarin [79], sulforaphane [80], Hb pretreatment [81], melatonin [82], and recombinant human erythropoietin [83], calycosin [84], (-)-epicatechin [67], luteolin [85], and ghrelin [86].

5.1.2. Peroxisome Proliferator-Activated Receptor (PPAR) $\gamma$ Signaling. PPAR $\gamma$ regulates CAT expression and is another antioxidant signaling pathway. CAT is ubiquitously expressed in all cell types including glia and neurons and is predominantly localized in peroxisomes. A PPAR response element is present in the CAT gene promoter, indicating a direct regulatory interaction. 15 -Deoxy- $\Delta 12,14$-prostaglandin J2 (15d-PGJ2) is a nonenzymatic breakdown product of prostaglandin D2; unlike synthetic thiazolidinediones, 15dPGJ2 acts as an endogenous ligand for PPAR $\gamma$ to promote the expression of CAT, which was shown to be associated with decreased inflammation, oxidative damage, and neuronal loss in a rat model of $\mathrm{ICH}$ [87]. It was noted in the cerebral hemorrhage model that telmisartan can induce the expression of receptor $\gamma$ activated by endothelial nitric oxide synthase and peroxisome proliferators and reduce oxidative stress, apoptosis signals, and tumor necrosis factor- $\alpha$ and cyclooxygenase- 2 expression [88].

\subsection{Decreased ROS Production following ICH}

5.2.1. NOX. The inhibition of NOX reduces the generation of endogenous ROS. A small ubiquitin-related modifier was shown to negatively regulate NOX5 in human neutrophils and vascular smooth muscle cells, thus limiting the production of ROS. However, there is little known about the involvement of small ubiquitin-related modifiers in $\mathrm{ICH}$ [89]. Melatonin was previously found to inhibit ROS 


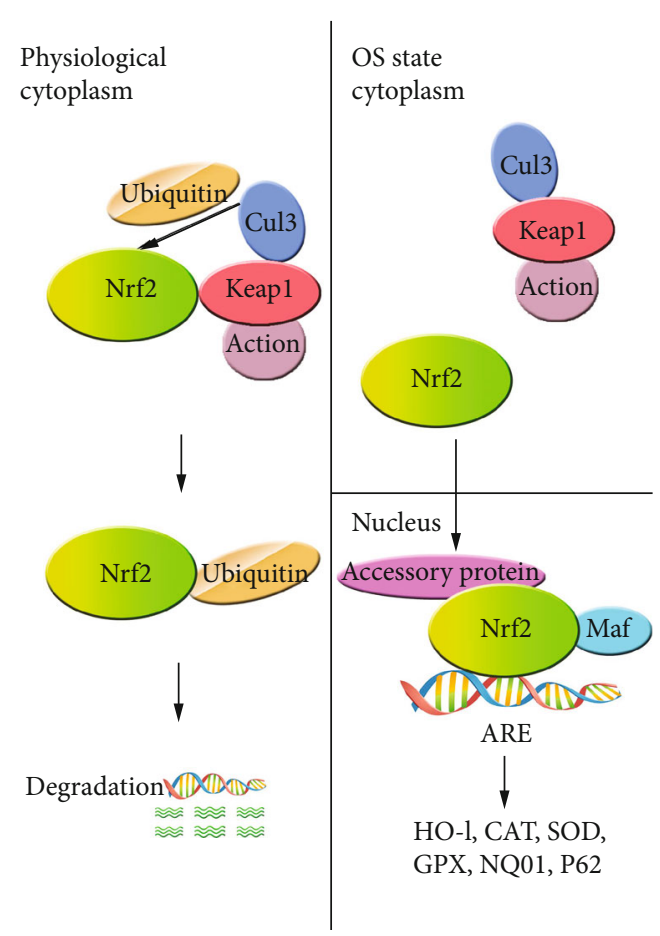

FIgURe 3: Description of antioxidant enzyme system regulation via the Keap1-Nrf2-ARE pathway. (a) Under normal basal conditions, Keap1 binds Nrf2 and keeps its level low by ubiquitination and proteasomal degradation; (b) under OS conditions, Keap1 is oxidized by OS, and dissociation of Nrf2 from Keap1 enables Nrf2 to translocate to the nucleus. Nrf2 combines with the small Maf protein to form a Nrf2-Maf heterodimer, and Nrf2 binds to accessory protein and then ARE activates gene expression of HO1, NQO1, GPX, SOD, CAT, and the autophagy protein p62. ARE: antioxidant response element; CAT: catalase; Cul3: Cullin3; GPX: glutathione peroxidase; HO-1: heme oxygenase-1; ICH: intracerebral hemorrhage; Keap1: Kelch-like ECH-associated protein 1; Maf: musculoaponeurotic fibrosarcoma; NQO1: NAPDH quinone oxidoreductase 1; Nrf2: nuclear factor erythroid 2-related factor 2; OS: oxidative stress; SOD: superoxide dismutase.

generation and OS after ICH [73]. Meanwhile, overexpression of the ubiquitin ligase ring finger (RNF) 34 exacerbated brain injury after ICH by promoting peroxisome proliferator-activated receptor coactivator- (PGC-) $1 \alpha$ degradation while stimulating the generation of mitochondrial ROS. Thus, genetic ablation of RNF34 is a potential strategy for the treatment of ICH [90]. The NADPH oxidase inhibitor apocynin improved the therapeutic efficacy of mesenchymal stem cells in the acute stage of ICH by exerting neuroprotective effects and enhancing the integrity of cerebral vasculature [12].

5.2.2. Mitochondria. Sodium benzoate was reported to mitigate OS-induced secondary brain injury, inhibit neuronal apoptosis, and suppress ROS production in mitochondria after $\mathrm{ICH}$ via $\mathrm{DJ}-1 /$ protein kinase $\mathrm{B}$ (AKT)/I $\kappa \mathrm{B}$ kinase $(\mathrm{IKK}) / \mathrm{NF}-\kappa \mathrm{B}$ signaling [91]. The alleviation of neurologic deficits by deferoxamine via inhibition of PGC- $1 \alpha$ signaling was shown to reduce OS caused by mitochondrial dysfunction [92]. Besides, drugs that can reduce the oxidative stress induced by mitochondrial function damage in $\mathrm{ICH}$ animal models include Dexmedetomidine (Dex) [92], Pyrroloquinoline Quinone (PQQ) [93], and melatonin [73].

5.3. Elimination of ROS following ICH. Intracerebroventricular injection of recombinant $\alpha 1$-microglobulin (A1M) resulted in its coexistence with extracellular $\mathrm{Hb}$, while injection of human $\mathrm{A} 1 \mathrm{M}$ mitigated the inflammatory response and mitochondrial damage in a rabbit model of IVH [94]. As a radical scavenger, A1M eliminates both heme and radicals, thus providing early protection to the immature brain in preterm IVH [94]. The novel free radical scavenger NSP-116 was found to alter cerebral blood flow and alleviate neurologic deficits in a model of I/R injury caused by middle cerebral artery occlusion; it also suppressed the expansion of hematomas and reduced neurologic deficits [95]. Edaravone, a free radical scavenger, reduced cerebral edema, and lipid peroxidation following IVH in rats and repeated administration improved learning and memory performance [96]. Hydrogen gas was found to reduce OS damage by eliminating $\mathrm{OH}-$ in a rat model of cerebral I/R [97], and inhaled hydrogen diminishes OS and brain edema $24 \mathrm{~h}$ after ICH, although it did not improve clinical outcome [98]. In addition, Glibenclamide (GLI) [99] and tempol [100] are also free radical scavengers for oxidative stress after $\mathrm{ICH}$.

5.4. Effects of Antioxidants following ICH. Melatonin ( $\mathrm{N}$-acetyl-5-methoxytryptamine) is an indolamine that is primarily synthesized by the pineal gland and can easily pass through the BBB. Melatonin improved severe $\mathrm{ICH}$-induced brain injury by mitigating OS, apoptosis, inflammation, DNA damage, brain edema, and $\mathrm{BBB}$ damage and by inhibiting MPTP opening [73], and in a SAH model, it mitigated cerebral OS by increasing the expression of HO-1, NQO1, $\mathrm{NADPH}$, and GST- $\alpha 1$, possibly via activation of the Nrf2/ARE signaling pathway [82]. Baicalein treatment reduced OS in rats by increasing the activity of SOD and GPX while downregulating the expression of MDA in the brain. Thus, baicalein can potentially be used to treat $\mathrm{ICH}$ and related brain injuries [101]. Danhong-a traditional Chinese medicine extracted from 2 herbs (Salviae miltiorrhiza Bunge (Danshen, China) and Carthamus tinctorius L (Honghua, China))-contains flavonoids and phenolic compounds and was reported to increase the expression of peroxiredoxin (Prx) 1 in astrocytes, thereby preventing severe brain injury following ICH in aged rats [102]. In addition, carnosine [103], COA-Cl (a novel synthesized nucleoside analog) [104], AE1-259-01 EP2 receptor agonists [105], green tea and red tea [57], protocatechuic acid (PCA) [106], nebivolol [107], 14Adiponectin (APN) [108], metformin [109], C1q/tumor necrosis factor-related protein 3 (CTRPs) [15], gastrodin [110], Naringin (NGN) [111], and Parthenolide (PN) [112] are all effective antioxidants in ICH animal models.

\section{Summary and Outlook}

The pathophysiologic processes that occur after ICH are complex, involving the neuroinflammatory response, OS, 
cytotoxicity caused by erythrocyte lysis, and the production of thrombin. Elucidating the causes of brain injury and the underlying molecular mechanisms and identifying novel markers of OS in the context of ICH will enable the development of effective interventions for the prevention and treatment of secondary brain injury following $\mathrm{ICH}$.

\section{Data Availability}

The data that support the findings of this study are openly available in PubMed at https://pubmed.ncbi.nlm.nih.gov/. Data sharing is not applicable to this article as no new data were created or analyzed in this study.

\section{Disclosure}

The funders had no role in decision to publish or preparation of the manuscript.

\section{Conflicts of Interest}

The authors declare no conflict of interest.

\section{Acknowledgments}

This research was funded by the National Natural Science Foundation of China, grant number 81771294.

\section{Supplementary Materials}

Table 1: potential Nrf2 agonists against oxidative stress in ICH. Table 2: the potential therapy targets at the oxidative stress caused by ICH. (Supplementary Materials)

\section{References}

[1] F. Siaw-Debrah, M. Nyanzu, H. Ni et al., "Preclinical studies and translational applications of intracerebral hemorrhage," BioMed Research International, vol. 2017, Article ID 5135429, 18 pages, 2017.

[2] J. F. Clark, M. Loftspring, W. L. Wurster et al., "Bilirubin oxidation products, oxidative stress, and intracerebral hemorrhage," Acta Neurochirurgica. Supplement, vol. 105, pp. 712, 2008.

[3] J. W. Park, J. E. Kim, M. J. Kang et al., "Anti-oxidant activity of gallotannin-enriched extract of Galla Rhois can associate with the protection of the cognitive impairment through the regulation of BDNF signaling pathway and neuronal cell function in the scopolamine-treated ICR mice," Antioxidants, vol. 8, no. 10, p. 450, 2019.

[4] Y. Zhang, M. Xu, C. Hu et al., "Sargassum fusiforme fucoidan SP2 extends the lifespan of Drosophila melanogaster by upregulating the Nrf2-mediated antioxidant signaling pathway," Oxidative Medicine and Cellular Longevity, vol. 2019, Article ID 8918914, 15 pages, 2019.

[5] D. P. Jones, "Radical-free biology of oxidative stress," American Journal of Physiology. Cell Physiology, vol. 295, no. 4, pp. C849-C868, 2008.

[6] J. Zeng, Y. Chen, R. Ding et al., "Isoliquiritigenin alleviates early brain injury after experimental intracerebral hemorrhage via suppressing ROS- and/or NF- $\kappa \mathrm{B}$-mediated NLRP3 inflammasome activation by promoting Nrf2 antioxidant pathway," Journal of Neuroinflammation, vol. 14, no. 1, p. 119, 2017.

[7] H. Shang, D. Yang, W. Zhang et al., "Time course of Keap1Nrf2 pathway expression after experimental intracerebral haemorrhage: correlation with brain oedema and neurological deficit," Free Radical Research, vol. 47, no. 5, pp. 368375, 2013.

[8] L. Zhang, J. Wu, X. Duan et al., "NADPH oxidase: a potential target for treatment of stroke," Oxidative Medicine and Cellular Longevity, vol. 2016, Article ID 5026984, 9 pages, 2016.

[9] A. Coyoy, A. Valencia, A. Guemez-Gamboa, and J. Morán, "Role of NADPH oxidase in the apoptotic death of cultured cerebellar granule neurons," Free Radical Biology \& Medicine, vol. 45, no. 8, pp. 1056-1064, 2008.

[10] R. Reinehr, B. Görg, S. Becker et al., "Hypoosmotic swelling and ammonia increase oxidative stress by NADPH oxidase in cultured astrocytes and vital brain slices," Glia, vol. 55, no. 7, pp. 758-771, 2007.

[11] H. Sumimoto, K. Miyano, and R. Takeya, "Molecular composition and regulation of the Nox family NAD $(\mathrm{P}) \mathrm{H}$ oxidases," Biochemical and Biophysical Research Communications, vol. 338, no. 1, pp. 677-686, 2005.

[12] S. Min, O. Kim, J. Bae, and T. Chung, "Effect of pretreatment with the NADPH oxidase inhibitor apocynin on the therapeutic efficacy of human placenta-derived mesenchymal stem cells in intracerebral hemorrhage," International Journal of Molecular Sciences, vol. 19, no. 11, p. 3679, 2018.

[13] M. T. Zia, A. Csiszar, N. Labinskyy et al., "Oxidative-nitrosative stress in a rabbit pup model of germinal matrix hemorrhage: role of $\mathrm{NAD}(\mathrm{P}) \mathrm{H}$ oxidase," Stroke, vol. 40, no. 6, pp. 2191-2198, 2009.

[14] J. Tang, J. Liu, C. Zhou et al., "Role of NADPH oxidase in the brain injury of intracerebral hemorrhage," Journal of Neurochemistry, vol. 94, no. 5, pp. 1342-1350, 2005.

[15] B. Yang, S. Wang, S. Yu et al., "C1q/tumor necrosis factorrelated protein 3 inhibits oxidative stress during intracerebral hemorrhage via PKA signaling," Brain Research, vol. 1657, pp. 176-184, 2017.

[16] L. Feng, Y. Chen, R. Ding et al., "P2X7R blockade prevents NLRP3 inflammasome activation and brain injury in a rat model of intracerebral hemorrhage: involvement of peroxynitrite," Journal of Neuroinflammation, vol. 12, no. 1, p. 190, 2015.

[17] R. Zhang, M. L. Brennan, Z. Shen et al., "Myeloperoxidase functions as a major enzymatic catalyst for initiation of lipid peroxidation at sites of inflammation*," The Journal of Biological Chemistry, vol. 277, no. 48, pp. 46116-46122, 2002.

[18] J. K. Y. Tse, "Gut microbiota, nitric oxide, and microglia as prerequisites for neurodegenerative disorders," ACS Chemical Neuroscience, vol. 8, no. 7, pp. 1438-1447, 2017.

[19] A. Sarniak, J. Lipińska, K. Tytman, and S. Lipińska, "Endogenous mechanisms of reactive oxygen species (ROS) generation," Postẹpy Higieny i Medycyny Doświadczalnej (Online), vol. 70, pp. 1150-1165, 2016.

[20] F. L. Muller, Y. Liu, and H. Van Remmen, "Complex III releases superoxide to both sides of the inner mitochondrial membrane*," The Journal of Biological Chemistry, vol. 279, no. 47, pp. 49064-49073, 2004.

[21] M. D. Brand, “The sites and topology of mitochondrial superoxide production," Experimental Gerontology, vol. 45, no. 78, pp. 466-472, 2010. 
[22] J. Qu, W. Chen, R. Hu, and H. Feng, "The injury and therapy of reactive oxygen species in intracerebral hemorrhage looking at mitochondria," Oxidative Medicine and Cellular Longevity, vol. 2016, Article ID 2592935, 9 pages, 2016.

[23] Z. Z. Duan, F. Zhang, F. Y. Li et al., "Protease activated receptor 1 (PAR1) enhances Src-mediated tyrosine phosphorylation of NMDA receptor in intracerebral hemorrhage (ICH)," Scientific Reports, vol. 6, no. 1, p. 29246, 2016.

[24] Z. Jiao, X. Liu, Y. Ma et al., "Adipose-derived stem cells protect ischemia-reperfusion and partial hepatectomy by attenuating endoplasmic reticulum stress," Frontiers in Cell and Development Biology, vol. 8, p. 177, 2020.

[25] C. D. Ochoa, R. F. Wu, and L. S. Terada, "ROS signaling and ER stress in cardiovascular disease," Molecular Aspects of Medicine, vol. 63, pp. 18-29, 2018.

[26] S. S. Cao and R. J. Kaufman, "Endoplasmic reticulum stress and oxidative stress in cell fate decision and human disease," Antioxidants \& Redox Signaling, vol. 21, no. 3, pp. 396-413, 2014.

[27] M. Niu, X. Dai, W. Zou et al., “Autophagy, endoplasmic reticulum stress and the unfolded protein response in intracerebral hemorrhage," Translational Neuroscience, vol. 8, pp. 37-48, 2017.

[28] J. Aronowski and X. Zhao, "Molecular pathophysiology of cerebral hemorrhage: secondary brain injury," Stroke, vol. 42, no. 6, pp. 1781-1786, 2011.

[29] M. Katsu, K. Niizuma, H. Yoshioka, N. Okami, H. Sakata, and P. H. Chan, "Hemoglobin-induced oxidative stress contributes to matrix metalloproteinase activation and blood-brain barrier dysfunction in vivo," Journal of Cerebral Blood Flow and Metabolism, vol. 30, no. 12, pp. 1939-1950, 2010.

[30] Y. Gu and S. Yang, "Effects of hemoglobin on the expression and distribution of inducible nitric oxide synthase after intracerebral hemorrhage," Zhonghua Yi Xue Za Zhi, vol. 94, no. 36, pp. 2857-2860, 2014.

[31] S. Yang, Y. Chen, X. Deng et al., "Hemoglobin-induced nitric oxide synthase overexpression and nitric oxide production contribute to blood-brain barrier disruption in the rat," Journal of Molecular Neuroscience, vol. 51, no. 2, pp. 352-363, 2013.

[32] J. M. Gutteridge and A. Smith, "Antioxidant protection by haemopexin of haem-stimulated lipid peroxidation," The Biochemical Journal, vol. 256, no. 3, pp. 861-865, 1988.

[33] R. F. Regan, J. Chen, and L. Benvenisti-Zarom, "Heme oxygenase- 2 gene deletion attenuates oxidative stress in neurons exposed to extracellular hemin," BMC Neuroscience, vol. 5, no. 1, p. 34, 2004.

[34] K. A. Hanafy, J. A. Gomes, and M. Selim, "Rationale and current evidence for testing iron chelators for treating stroke," Current Cardiology Reports, vol. 21, no. 4, p. 20, 2019.

[35] J. Cadet, S. Loft, R. Olinski et al., "Biologically relevant oxidants and terminology, classification and nomenclature of oxidatively generated damage to nucleobases and 2deoxyribose in nucleic acids," Free Radical Research, vol. 46, no. 4, pp. 367-381, 2012.

[36] C. L. Hawkins and M. J. Davies, "Detection, identification, and quantification of oxidative protein modifications," The Journal of Biological Chemistry, vol. 294, no. 51, pp. 1968319708, 2019.

[37] A. Cipak Gasparovic, N. Zarkovic, K. Zarkovic et al., "Biomarkers of oxidative and nitro-oxidative stress: conventional and novel approaches," British Journal of Pharmacology, vol. 174, no. 12, pp. 1771-1783, 2017.

[38] T. Jiang, B. Harder, M. Rojo de la Vega, P. K. Wong, E. Chapman, and D. D. Zhang, "p62 links autophagy and Nrf2 signaling," Free Radical Biology and Medicine, vol. 88, no. Part B, pp. 199-204, 2015.

[39] R. Scherz-Shouval, E. Shvets, E. Fass, H. Shorer, L. Gil, and Z. Elazar, "Reactive oxygen species are essential for autophagy and specifically regulate the activity of Atg4," The EMBO Journal, vol. 38, no. 10, 2019.

[40] G. Filomeni, D. De Zio, and F. Cecconi, "Oxidative stress and autophagy: the clash between damage and metabolic needs," Cell Death and Differentiation, vol. 22, no. 3, pp. 377-388, 2015.

[41] X. Shen, L. Ma, W. Dong et al., "Autophagy regulates intracerebral hemorrhage induced neural damage via apoptosis and NF- $\kappa$ B pathway," Neurochemistry International, vol. 96, pp. 100-112, 2016.

[42] Y. Fang, S. Chen, C. Reis, and J. Zhang, "The role of autophagy in subarachnoid hemorrhage: an update," Current Neuropharmacology, vol. 16, no. 9, pp. 1255-1266, 2018.

[43] G. Pistritto, D. Trisciuoglio, C. Ceci, A. Garufi, and G. D'Orazi, “Apoptosis as anticancer mechanism: function and dysfunction of its modulators and targeted therapeutic strategies," Aging (Albany NY), vol. 8, no. 4, pp. 603-619, 2016.

[44] C. Yang, K. E. Hawkins, S. Doré, and E. Candelario-Jalil, "Neuroinflammatory mechanisms of blood-brain barrier damage in ischemic stroke," American Journal of Physiology. Cell Physiology, vol. 316, no. 2, pp. C135-C153, 2019.

[45] W. Abdullahi, D. Tripathi, and P. T. Ronaldson, "Blood-brain barrier dysfunction in ischemic stroke: targeting tight junctions and transporters for vascular protection," American Journal of Physiology. Cell Physiology, vol. 315, no. 3, pp. C343-c356, 2018.

[46] J. Zheng, L. Shi, F. Liang et al., "Sirt3 ameliorates oxidative stress and mitochondrial dysfunction after intracerebral hemorrhage in diabetic rats," Frontiers in Neuroscience, vol. 12, p. 414, 2018.

[47] L. Fialkow, Y. Wang, and G. P. Downey, "Reactive oxygen and nitrogen species as signaling molecules regulating neutrophil function," Free Radical Biology \& Medicine, vol. 42, no. 2, pp. 153-164, 2007.

[48] G. J. Maghzal, K. M. Cergol, S. R. Shengule et al., "Assessment of myeloperoxidase activity by the conversion of hydroethidine to 2-chloroethidium*," The Journal of Biological Chemistry, vol. 289, no. 9, pp. 5580-5595, 2014.

[49] J. Cadet, K. J. A. Davies, M. H. G. Medeiros, P. di Mascio, and J. R. Wagner, "Formation and repair of oxidatively generated damage in cellular DNA," Free Radical Biology \& Medicine, vol. 107, pp. 13-34, 2017.

[50] T. Nakamura, R. F. Keep, Y. Hua, J. T. Hoff, and G. Xi, “Oxidative DNA injury after experimental intracerebral hemorrhage," Brain Research, vol. 1039, no. 1-2, pp. 30-36, 2005.

[51] Y. C. Chen, C. M. Chen, J. L. Liu, S. T. Chen, M. L. Cheng, and D. T. Y. Chiu, "Oxidative markers in spontaneous intracerebral hemorrhage: leukocyte 8-hydroxy-2'-deoxyguanosine as an independent predictor of the 30-day outcome," Journal of Neurosurgery, vol. 115, no. 6, pp. 1184-1190, 2011.

[52] L. Lorente, M. M. Martín, A. F. González-Rivero et al., "High serum DNA and RNA oxidative damage in non-surviving 
patients with spontaneous intracerebral hemorrhage," Neurocritical Care, vol. 33, no. 1, pp. 90-96, 2020.

[53] H. F. Moselhy, R. G. Reid, S. Yousef, and S. P. Boyle, "A specific, accurate, and sensitive measure of total plasma malondialdehyde by HPLC," Journal of Lipid Research, vol. 54, no. 3, pp. 852-858, 2013.

[54] L. Lorente, M. M. Martín, P. Abreu-González et al., "Serum malondialdehyde levels and mortality in patients with spontaneous intracerebral hemorrhage," World Neurosurgery, vol. 113, pp. e542-e547, 2018.

[55] Q. Wu, X. S. Zhang, H. D. Wang et al., “Astaxanthin activates nuclear factor erythroid-related factor 2 and the antioxidant responsive element (Nrf2-ARE) pathway in the brain after subarachnoid hemorrhage in rats and attenuates early brain injury," Marine Drugs, vol. 12, no. 12, pp. 6125-6141, 2014.

[56] C. C. Wei, Y. Y. Kong, G. Q. Li, Y. F. Guan, P. Wang, and C. Y. Miao, "Nicotinamide mononucleotide attenuates brain injury after intracerebral hemorrhage by activating Nrf2/HO-1 signaling pathway," Scientific Reports, vol. 7, no. 1, p. 717, 2017.

[57] P. M. Sosa, M. A. de Souza, and P. B. Mello-Carpes, "Green tea and red tea from Camellia sinensis partially prevented the motor deficits and striatal oxidative damage induced by hemorrhagic stroke in rats," Neural Plasticity, vol. 2018, Article ID 5158724, 8 pages, 2018.

[58] S. Duan, F. Wang, J. Cao, and C. Wang, "Exosomes derived from microRNA-146a-5p-enriched bone marrow mesenchymal stem cells alleviate intracerebral hemorrhage by inhibiting neuronal apoptosis and microglial M1 polarization," Drug Design, Development and Therapy, vol. Volume 14, pp. 3143-3158, 2020.

[59] C. M. Spickett, "The lipid peroxidation product 4-hydroxy-2nonenal: advances in chemistry and analysis," Redox Biology, vol. 1, no. 1, pp. 145-152, 2013.

[60] M. L. Selley, "Determination of the lipid peroxidation product (E)-4-hydroxy-2-nonenal in clinical samples by gas chromatography-negative-ion chemical ionisation mass spectrometry of the O-pentafluorobenzyl oxime," Journal of Chromatography. B, Biomedical Sciences and Applications, vol. 691, no. 2, pp. 263-268, 1997.

[61] I. Jarocka-Karpowicz, A. Syta-Krzyżanowska, J. Kochanowicz, and Z. D. Mariak, "Clinical prognosis for SAH consistent with redox imbalance and lipid peroxidation," Molecules, vol. 25, no. 8, p. 1921, 2020.

[62] D. Il'yasova, J. D. Morrow, A. Ivanova, and L. E. Wagenknecht, "Epidemiological marker for oxidant status: comparison of the ELISA and the gas chromatography/mass spectrometry assay for urine 2,3-dinor-5,6-dihydro- $15-\mathrm{F}_{2 \mathrm{t}}{ }^{-}$ isoprostane," Annals of Epidemiology, vol. 14, no. 10, pp. 793-797, 2004.

[63] Q. du, W. H. Yu, X. Q. Dong et al., "Plasma 8-isoprostaglandin F2 $\alpha$ concentrations and outcomes after acute intracerebral hemorrhage," Clinica Chimica Acta, vol. 437, pp. 141-146, 2014.

[64] T. B. Corcoran, E. Mas, A. E. Barden et al., "Are isofurans and neuroprostanes increased after subarachnoid hemorrhage and traumatic brain injury?," Antioxidants \& Redox Signaling, vol. 15, no. 10, pp. 2663-2667, 2011.

[65] M. Zheng, X. Wang, J. Yang, S. Ma, Y. Wei, and S. Liu, "Changes of complement and oxidative stress parameters in patients with acute cerebral infarction or cerebral hemor- rhage and the clinical significance," Experimental and Therapeutic Medicine, vol. 19, no. 1, pp. 703-709, 2020.

[66] Z. Wang, S. Guo, J. Wang, Y. Shen, J. Zhang, and Q. Wu, "Nrf2/HO-1 mediates the neuroprotective effect of mangiferin on early brain injury after subarachnoid hemorrhage by attenuating mitochondria-related apoptosis and neuroinflammation," Scientific Reports, vol. 7, no. 1, p. 11883, 2017.

[67] X. Lan, X. Han, Q. Li, and J. Wang, “(-)-Epicatechin, a natural flavonoid compound, protects astrocytes against hemoglobin toxicity via Nrf2 and AP-1 signaling pathways," Molecular Neurobiology, vol. 54, no. 10, pp. 7898-7907, 2017.

[68] T. Nybo, M. J. Davies, and A. Rogowska-Wrzesinska, "Analysis of protein chlorination by mass spectrometry," Redox Biology, vol. 26, p. 101236, 2019.

[69] G. R. Zheng, B. Chen, J. Shen et al., "Serum myeloperoxidase concentrations for outcome prediction in acute intracerebral hemorrhage," Clinica Chimica Acta, vol. 487, pp. 330-336, 2018.

[70] D. Giustarini, D. Tsikas, G. Colombo et al., "Pitfalls in the analysis of the physiological antioxidant glutathione (GSH) and its disulfide (GSSG) in biological samples: an elephant in the room," Journal of Chromatography. B, Analytical Technologies in the Biomedical and Life Sciences, vol. 1019, pp. 2128, 2016.

[71] J. Gruber, S. Y. Tang, A. M. Jenner et al., “Allantoin in human plasma, serum, and nasal-lining fluids as a biomarker of oxidative stress: avoiding artifacts and establishing real in vivo concentrations," Antioxidants \& Redox Signaling, vol. 11, no. 8, pp. 1767-1776, 2009.

[72] S. Q. Qian, X. C. Hu, S. R. He, B. B. Li, X. D. Zheng, and G. H. Pan, "Prognostic value of serum thioredoxin concentrations after intracerebral hemorrhage," Clinica Chimica Acta, vol. 455, pp. 15-19, 2016.

[73] Z. Wang, F. Zhou, Y. Dou et al., "Melatonin alleviates intracerebral hemorrhage-induced secondary brain injury in rats via suppressing apoptosis, inflammation, oxidative stress, DNA damage, and mitochondria injury," Translational Stroke Research, vol. 9, no. 1, pp. 74-91, 2018.

[74] X. Zhao, G. Sun, J. Zhang et al., “Transcription factor Nrf2 protects the brain from damage produced by intracerebral hemorrhage," Stroke, vol. 38, no. 12, pp. 3280-3286, 2007.

[75] S. Petri, S. Körner, and M. Kiaei, "Nrf2/ARE signaling pathway: key mediator in oxidative stress and potential therapeutic target in ALS," Neurology Research International, vol. 2012, Article ID 878030, 7 pages, 2012.

[76] C. Y. Zhang, X. M. Ren, H. B. Li et al., "Simvastatin alleviates inflammation and oxidative stress in rats with cerebral hemorrhage through Nrf2-ARE signaling pathway," European Review for Medical and Pharmacological Sciences, vol. 23, no. 14, pp. 6321-6329, 2019.

[77] Y. Y. Shi, H. F. Cui, and B. J. Qin, "Monomethyl fumarate protects cerebral hemorrhage injury in rats via activating microRNA-139/Nrf2 axis," European Review for Medical and Pharmacological Sciences, vol. 23, no. 11, pp. 50125019, 2019.

[78] T. Sugiyama, T. Imai, S. Nakamura et al., "A novel Nrf2 activator, RS9, attenuates secondary brain injury after intracerebral hemorrhage in sub-acute phase," Brain Research, vol. 1701, pp. 137-145, 2018.

[79] R. Yuan, H. Fan, S. Cheng et al., "Silymarin prevents NLRP3 inflammasome activation and protects against intracerebral 
hemorrhage," Biomedicine \& Pharmacotherapy, vol. 93, pp. 308-315, 2017.

[80] G. Chen, Q. Fang, J. Zhang, D. Zhou, and Z. Wang, "Role of the Nrf2-ARE pathway in early brain injury after experimental subarachnoid hemorrhage," Journal of Neuroscience Research, vol. 89, no. 4, pp. 515-523, 2011.

[81] Y. Yang, Z. Xi, Y. Xue et al., "Hemoglobin pretreatment endows rat cortical astrocytes resistance to hemin- induced toxicity via Nrf2/HO-1 pathway," Experimental Cell Research, vol. 361, no. 2, pp. 217-224, 2017.

[82] Z. Wang, C. Ma, C. J. Meng et al., "Melatonin activates the Nrf2-ARE pathway when it protects against early brain injury in a subarachnoid hemorrhage model," Journal of Pineal Research, vol. 53, no. 2, pp. 129-137, 2012.

[83] J. Zhang, Y. Zhu, D. Zhou, Z. Wang, and G. Chen, "Recombinant human erythropoietin (rhEPO) alleviates early brain injury following subarachnoid hemorrhage in rats: possible involvement of Nrf2-ARE pathway," Cytokine, vol. 52, no. 3, pp. 252-257, 2010.

[84] C. Chen, J. Cui, X. Ji, and L. Yao, "Neuroprotective functions of calycosin against intracerebral hemorrhage-induced oxidative stress and neuroinflammation," Future Medicinal Chemistry, vol. 12, no. 7, pp. 583-592, 2020.

[85] X. Tan, Y. Yang, J. Xu et al., "Luteolin exerts neuroprotection via modulation of the p62/Keap1/Nrf2 pathway in intracerebral hemorrhage," Frontiers in Pharmacology, vol. 10, p. 1551, 2020.

[86] Y. Cheng, B. Chen, W. Xie et al., "Ghrelin attenuates secondary brain injury following intracerebral hemorrhage by inhibiting NLRP3 inflammasome activation and promoting Nrf2/ARE signaling pathway in mice," International Immunopharmacology, vol. 79, p. 106180, 2020.

[87] X. Zhao, Y. Zhang, R. Strong, J. C. Grotta, and J. Aronowski, "15d-Prostaglandin J2Activates peroxisome proliferatoractivated Receptor- $\gamma$, promotes expression of catalase, and reduces inflammation, behavioral dysfunction, and neuronal loss after intracerebral hemorrhage in rats," Journal of Cerebral Blood Flow and Metabolism, vol. 26, no. 6, pp. 811820, 2005.

[88] K. H. Jung, K. Chu, S. T. Lee et al., "Blockade of AT1 receptor reduces apoptosis, inflammation, and oxidative stress in normotensive rats with intracerebral hemorrhage," The Journal of Pharmacology and Experimental Therapeutics, vol. 322, no. 3, pp. 1051-1058, 2007.

[89] D. Pandey, F. Chen, A. Patel et al., "SUMO1 negatively regulates reactive oxygen species production from NADPH oxidases," Arteriosclerosis, Thrombosis, and Vascular Biology, vol. 31, no. 7, pp. 1634-1642, 2011.

[90] X. Qu, N. Wang, W. Chen, M. Qi, Y. Xue, and W. Cheng, "RNF34 overexpression exacerbates neurological deficits and brain injury in a mouse model of intracerebral hemorrhage by potentiating mitochondrial dysfunction-mediated oxidative stress," Scientific Reports, vol. 9, no. 1, p. 16296, 2019.

[91] W. Xu, T. Li, L. Gao et al., "Sodium benzoate attenuates secondary brain injury by inhibiting neuronal apoptosis and reducing mitochondria-mediated oxidative stress in a rat model of intracerebral hemorrhage: possible involvement of DJ-1/Akt/IKK/NF $\kappa B$ pathway," Frontiers in Molecular Neuroscience, vol. 12, p. 105, 2019.

[92] J. Huang and Q. Jiang, "Dexmedetomidine protects against neurological dysfunction in a mouse intracerebral hemor- rhage model by inhibiting mitochondrial dysfunctionderived oxidative stress," Journal of Stroke and Cerebrovascular Diseases, vol. 28, no. 5, pp. 1281-1289, 2019.

[93] H. Lu, J. Shen, X. Song et al., "Protective effect of pyrroloquinoline quinone (PQQ) in rat model of intracerebral hemorrhage," Cellular and Molecular Neurobiology, vol. 35, no. 7, pp. 921-930, 2015.

[94] O. Romantsik, A. A. Agyemang, S. Sveinsdóttir et al., "The heme and radical scavenger $\alpha 1$-microglobulin (A1M) confers early protection of the immature brain following preterm intraventricular hemorrhage," Journal of Neuroinflammation, vol. 16, no. 1, p. 122, 2019.

[95] T. Imai, S. Iwata, D. Miyo, S. Nakamura, M. Shimazawa, and H. Hara, "A novel free radical scavenger, NSP-116, ameliorated the brain injury in both ischemic and hemorrhagic stroke models," Journal of Pharmacological Sciences, vol. 141, no. 3, pp. 119-126, 2019.

[96] Z. Chen, J. Zhang, Q. Chen, J. Guo, G. Zhu, and H. Feng, "Neuroprotective effects of edaravone after intraventricular hemorrhage in rats," Neuroreport, vol. 25, no. 9, pp. 635640, 2014.

[97] I. Ohsawa, M. Ishikawa, K. Takahashi et al., "Hydrogen acts as a therapeutic antioxidant by selectively reducing cytotoxic oxygen radicals," Nature Medicine, vol. 13, no. 6, pp. 688694, 2007.

[98] A. Manaenko, T. Lekic, Q. Ma, R. P. Ostrowski, J. H. Zhang, and J. Tang, "Hydrogen inhalation is neuroprotective and improves functional outcomes in mice after intracerebral hemorrhage," Acta Neurochirurgica. Supplement, vol. 111, pp. 179-183, 2011.

[99] F. Zhou, Y. Liu, B. Yang, and Z. Hu, "Neuroprotective potential of glibenclamide is mediated by antioxidant and antiapoptotic pathways in intracerebral hemorrhage," Brain Research Bulletin, vol. 142, pp. 18-24, 2018.

[100] Y. Wanyong, T. Zefeng, X. Xiufeng et al., “Tempol alleviates intracerebral hemorrhage-induced brain injury possibly by attenuating nitrative stress," Neuroreport, vol. 26, no. 14, pp. 842-849, 2015.

[101] N. Wei, Y. Wei, B. Li, and L. Pang, "Baicalein promotes neuronal and behavioral recovery after Intracerebral hemorrhage via suppressing apoptosis, oxidative stress and neuroinflammation," Neurochemical Research, vol. 42, no. 5, pp. 13451353, 2017.

[102] S. Wang, L. Yu, G. Sun et al., "Danhong injection protects hemorrhagic brain by increasing peroxiredoxin 1 in aged rats," Frontiers in Pharmacology, vol. 11, p. 346, 2020.

[103] R. X. Xie, D. W. Li, X. C. Liu et al., "Carnosine attenuates brain oxidative stress and apoptosis after intracerebral hemorrhage in rats," Neurochemical Research, vol. 42, no. 2, pp. 541-551, 2017.

[104] F. Lu, T. Nakamura, N. Okabe et al., "COA-Cl, a novel synthesized nucleoside analog, exerts neuroprotective effects in the acute phase of intracerebral hemorrhage," Journal of Stroke and Cerebrovascular Diseases, vol. 25, no. 11, pp. 2637-2643, 2016.

[105] H. Wu, T. Wu, X. Han et al., "Cerebroprotection by the neuronal PGE2 receptor EP2 after intracerebral hemorrhage in middle-aged mice," Journal of Cerebral Blood Flow and Metabolism, vol. 37, no. 1, pp. 39-51, 2017.

[106] Z. Xi, X. Hu, X. Chen et al., "Protocatechuic acid exerts protective effects via suppression of the P38/JNK- NF- $\kappa$ B 
signalling pathway in an experimental mouse model of intracerebral haemorrhage," European Journal of Pharmacology, vol. 854, pp. 128-138, 2019.

[107] M. A. Aladag, Y. Turkoz, H. Parlakpinar, and M. Gul, "Nebivolol attenuates cerebral vasospasm both by increasing endothelial nitric oxide and by decreasing oxidative stress in an experimental subarachnoid haemorrhage," British Journal of Neurosurgery, vol. 31, no. 4, pp. 439-445, 2016.

[108] S. Wang, D. Li, C. Huang et al., "Overexpression of adiponectin alleviates intracerebral hemorrhage-induced brain injury in rats via suppression of oxidative stress," Neuroscience Letters, vol. 681, pp. 110-116, 2018.

[109] B. Qi, L. Hu, L. Zhu et al., "Metformin attenuates neurological deficit after intracerebral hemorrhage by inhibiting apoptosis, oxidative stress and neuroinflammation in rats," Neurochemical Research, vol. 42, no. 10, pp. 2912-2920, 2017.

[110] X. C. Liu, C. Z. Wu, X. F. Hu et al., "Gastrodin attenuates neuronal apoptosis and neurological deficits after experimental intracerebral hemorrhage," Journal of Stroke and Cerebrovascular Diseases, vol. 29, no. 1, p. 104483, 2020.

[111] N. Singh, Y. Bansal, R. Bhandari et al., "Naringin reverses neurobehavioral and biochemical alterations in intracerebroventricular collagenase-induced intracerebral hemorrhage in rats," Pharmacology, vol. 100, no. 3-4, pp. 172-187, 2017.

[112] J. A. Wang, M. L. Tong, B. Zhao, G. Zhu, D. H. Xi, and J. P. Yang, "Parthenolide ameliorates intracerebral hemorrhageinduced brain injury in rats," Phytotherapy Research, vol. 34, no. 1, pp. 153-160, 2019. 